\title{
Study of nonlinear Alfvén waves in an electron-positron plasma with a three-dimensional electromagnetic particle code
}

\author{
J. Zhao \\ Department of Electronics and Information, Toyama University, Toyama 930, Japan \\ K. I. Nishikawa \\ Department of Physics and Astronomy, The University of Iowa, Iowa City, Iowa 52242 \\ J. I. Sakai \\ Department of Electronics and Information, Toyama University, Toyama 930, Japan \\ T. Neubert \\ Space Physics Research Laboratory, The University of Michigan, Ann Arbor, Michigan 48109
}

(Received 30 July 1993; accepted 23 September 1993)

\begin{abstract}
Results from three-dimensional (3-D) electromagnetic particle simulations of Alfvén waves generated by an electron beam in a nonrelativistic electron-positron plasma are presented. The results show that electrostatic modes are excited due to the beam instability. The bunches of the particles (electrons and positrons) caused by electrostatic waves are directly involved in the generation of Alfvén waves. The Alfvén waves propagate along the beam as damped solitons accelerating the background particles. The simulation results are in good agreement with theoretical analysis.
\end{abstract}

\section{INTRODUCTION}

It is widely thought that the nonlinear interaction of Alfvén waves in electron-positron plasmas is of prime importance in understanding emission processes of pulsars. Much attention has been paid to the problems of wave propagation in electron-positron plasmas. The wave characteristics are quite different from those of electron-ion plasmas, since the natural separation of time- and spatial scales associated with high- and low-frequency motions no longer exists. A number of authors have investigated the nonlinear propagation of the waves in electron-positron plasmas. These include Sakai and Kawata ${ }^{1}$ and Mikhailovskii et al., ${ }^{2}$ who described solitary Alfvén wave propagation paralleled to a constant magnetic field in relativistic electron-positron plasmas, and Stenflo et al. ${ }^{3}$ who derived a set of nonlinear equations governing coupling of electromagnetic radiation with cold electrostatic oscillations. Others studied such phenomena as two-dimensional (2-D) effects on Alfvén vortices in a strongly magnetized electronpositron plasma ${ }^{4}$ and instability of shear Alfvén waves caused by current gradients. ${ }^{5}$ Most work done in the past in this area has used theoretical analysis of Alfvén wave propagation.

Our goal in this study is to investigate the characteristics of the nonlinear interaction in a region which is far from the center of the pulsar and attempt to establish a clearer picture of the formation mechanism of Alfvén waves by performing simulations with a three-dimensional (3-D) electromagnetic and relativistic particle code ${ }^{6-9} \mathrm{We}$ have found that the bunches of particles (electrons and positrons) caused by electrostatic waves excited by the beam instability are directly involved in the generation of Alfvén waves. The Alfvén waves propagate along the beam with characteristics of damped solitons further accelerating background particles. The plan of the paper is as follows.
In Sec. II, we present a system model and parameters used in the simulation. In Sec. III, we discuss the beam instability and compare it with the simulation results. In Sec. IV, the presence of particle bunches is argued to generate the Alfven waves on the bases of simulation results and linear dispersion theory analysis. The Alfvén waves are found to propagate with damped soliton characteristics and to accelerate particles, as explained in Sec. V. We summarize our results in the last section.

\section{SIMULATION MODEL AND PARAMETERS}

The code is a 3-D, fully electromagnetic and relativistic particle-in-cell (PIC) code. The system size used for the simulations is $L_{x}=L_{y}=85 \Delta, L_{z}=160 \Delta$ with two million particles, where $L_{x}, L_{y}$, and $L_{z}$ are the lengths of the system in the three dimensions and $\Delta(=1)$ is the grid size. Periodic boundary conditions are used for particles and fields in the $z$ direction, while radiating boundary conditions ${ }^{10}$ are used in the $x$ and $y$ directions. Particles which hit the boundaries are arrested there and then redistributed uniformly by having the boundaries slightly conducting. This scheme is possible for electron-positron simulations because the flux to the boundaries of electrons and positrons are comparable.

The particles are initially distributed homogeneously in the system. The electron beam is in a column with radius, $r_{\mathrm{eb}}=4.47 \Delta$, and the axis located at the center of the domain $\left(x_{\mathrm{cnt}}=y_{\mathrm{cnt}}=43\right)$ oriented along the $z$ direction. The plasma density inside the column is the same as the outside one and the column is initially charge neutral. Within the column, half of the electrons are beam clectrons, while the other half are background electrons so that the beam electron density is about half of the total electron density. The beam electron temperature, $T_{b}=0.09 T_{g}$, where $T_{g}$ is the temperature of background electrons. Ini- 
tially only the beam electrons drift with $v_{d}=3.5 v_{t g}$, where $v_{t g}$ is the background electron thermal velocity. Other parameters are as follows: $m_{p} / m_{e}=1, T_{g} / T_{p}=1\left(T_{p}\right.$ is the positron temperature), $\left(\omega_{p e} \Delta t\right)=0.1, \omega_{c e} / \omega_{p e}=0.4$, $\beta=0.11, \lambda_{D}=0.469 \Delta, c / \omega_{p e}=5.00, c / v_{t g}=10.67$, the gyroradius of electron and positron is $\rho_{e}=\rho_{p}=1.17$. The magnetic field consistent with the current density $J_{z}$ generated by the beam electrons is included initially. ${ }^{9}$

\section{ELECTROSTATIC BEAM MODE}

When small-amplitude electrostatic perturbations along the magnetic field are considered, the equations of motion for positrons and electrons in the column are to first order

$$
\begin{aligned}
& m n_{0} \frac{\partial u_{1 p}}{\partial t}=e n_{0} E_{1}-\frac{\partial}{\partial z}\left(n_{1 p} k_{\mathrm{B}} T_{p}\right), \\
& \frac{1}{2} m n_{0} \frac{\partial u_{1 g}}{\partial t}=-\frac{1}{2} e n_{0} E_{1}-\frac{\partial}{\partial z}\left(n_{1 g} k_{\mathrm{B}} T_{g}\right), \\
& \frac{1}{2} m n_{0} \frac{\partial u_{1 b}}{\partial t}=-\frac{1}{2} e n_{0} E_{1}-\frac{\partial}{\partial z}\left(n_{1 b} k_{\mathrm{B}} T_{b}\right) .
\end{aligned}
$$

Here $m, e, T, n_{0}, k_{\mathrm{B}}$ are the mass, charge, temperature, equilibrium density of the particles, and the Boltzmann constant, respectively. Also, $u_{1}, n_{1}, E_{1}$ are the perturbed velocity, density, and electric field, respectively. The subscripts $p, g, b$ refer to positrons, background electrons and beam electrons, respectively. The factor $1 / 2$ in Eqs. (2) and (3) comes from the fact that the densities of beam electrons and background electrons are half of the positron density. With the usual plane wave analysis applied to Eqs. (1)-(3), the perturbed velocities are obtained

$$
\begin{aligned}
& u_{1 p}=\frac{i e E_{1}}{m \omega}+\frac{k_{\mathrm{B}} n_{1 p} T_{p} k}{m n_{0} \omega}, \\
& u_{1 g}=-\frac{i e E_{1}}{m \omega}+\frac{2 k_{\mathrm{B}} n_{1 g} T_{g} k}{m n_{0} \omega}, \\
& u_{1 b}=-\frac{i e E_{t}}{m\left(\omega-k v_{d}\right)}+\frac{2 k_{\mathrm{B}} n_{1 b} T_{b} k}{m n_{0}\left(\omega-k v_{d}\right)} .
\end{aligned}
$$

Assuming that the beam electrons are streaming along the $z$ dircction with the drift velocity $v_{d}$ given initially in our system, the continuity equations for the positrons and electrons are expressed as

$$
\begin{aligned}
& \frac{\partial n_{1 p}}{\partial t}+n_{0} \frac{\partial u_{1 p}}{\partial z}=0, \\
& \frac{\partial n_{1 g}}{\partial t}+\frac{1}{2} n_{0} \frac{\partial u_{1 g}}{\partial z}=0, \\
& \frac{\partial n_{1 b}}{\partial t}+\frac{1}{2} n_{0} \frac{\partial u_{1 b}}{\partial z}+v_{d} \frac{\partial n_{1 b}}{\partial z}=0 .
\end{aligned}
$$

Performing the usual wave analysis and solving for the perturbed density, it yields

$$
n_{1 p}=k n_{0} u_{1 p} / \omega
$$
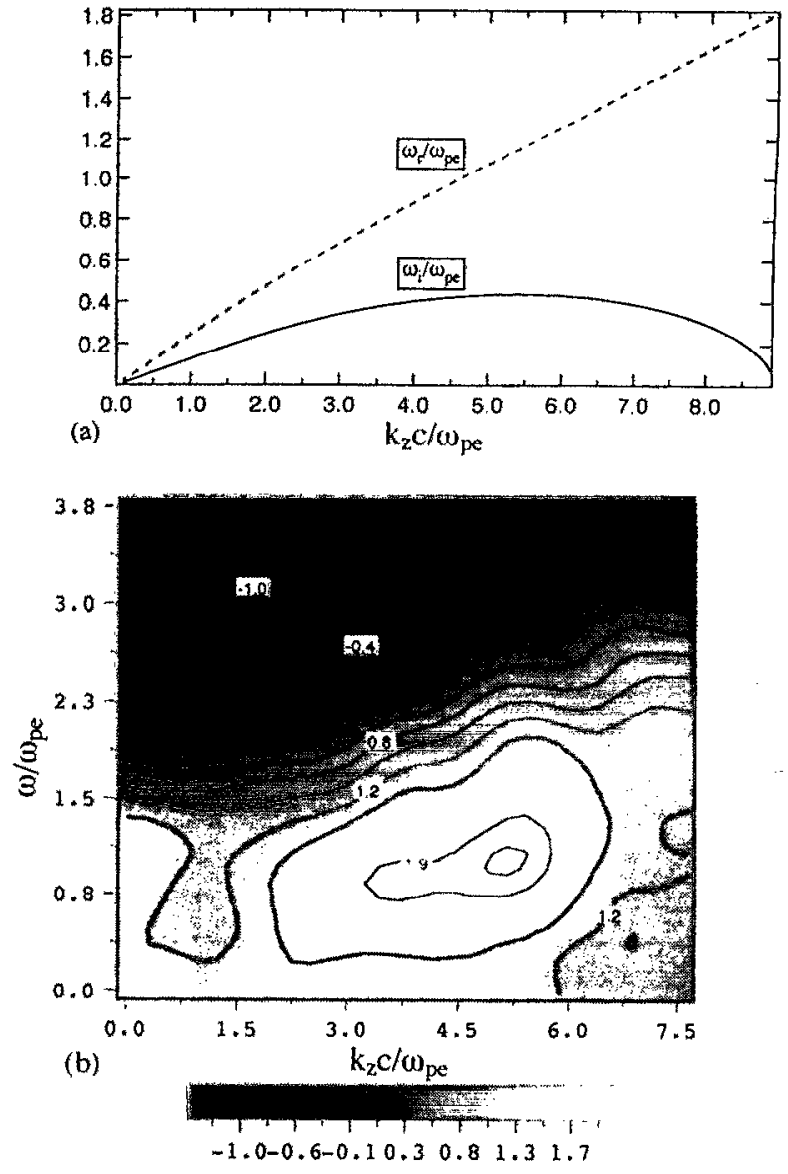

FIG. 1. (a) Dispersion relation of the waves excited by the beam instability. (b) Intensity of the charge density along the $z$ direction in the center of beam.

$$
\begin{aligned}
& n_{1 g}=k n_{0} u_{1 g} / 2 \omega, \\
& n_{1 b}=k n_{0} u_{1 b} / 2\left(\omega-k v_{d}\right) .
\end{aligned}
$$

Relating the charge density to the electric field, one arrives at the dispersion relation

$$
1=\frac{\omega_{p e}^{2}}{\omega^{2}-k^{2} v_{t p}^{2}}+\frac{\omega_{p e}^{2}}{2\left(\omega^{2}-k^{2} v_{t g}^{2}\right)}+\frac{\omega_{p e}^{2}}{2\left(\omega-k v_{d}\right)^{2}-2 k^{2} v_{t b}^{2}},
$$

where $v_{t p}, v_{t g}, v_{t b}$ are the thermal velocities of positrons, background electrons, and beam electrons, respectively. Equation (13) is fourth order in $\omega$ and can be solved for arbitrary values of $k$. The frequency will generally be complex $\left(\omega=\omega_{r}+i \omega_{i}\right)$, where $\omega_{i}>0$ corresponds to growing solutions and $\omega_{i}<0$ to damped solutions. With the simulation parameters, $v_{t p}=v_{t g}, v_{t b}=0.3 v_{t g}, v_{d}=3.5 v_{t g}$, we can find a branch that is unstable over a broad range of wave numbers. The dispersion relation for this branch is shown in Fig. 1(a). The plot of $k$ as function of $\omega_{r}$ (the real part of root) is the dispersion relation, while the $k$ at the maximum $\omega_{i}$ associates with the most unstable wave number.

Figure 1(b) shows intensity of the charge density obtained from the simulation results along the $z$ direction. The plot has been created by performing a 2-D Fourier 

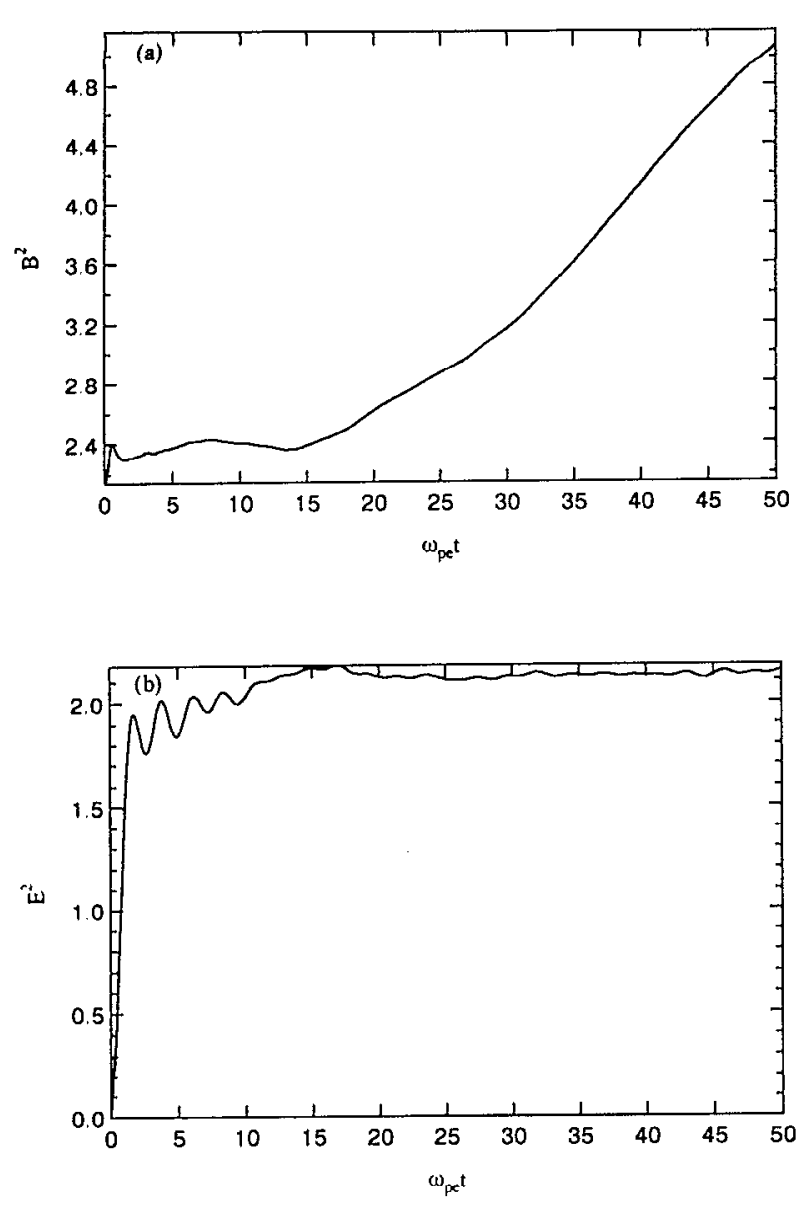

FIG. 2. The time cvolutions of electromagnetic field energies. (a) magnetic field energy; (b) electric field energy.

transformation (one space and one time) of the first 512 time steps. The gray scale is logarithmic in the wave intensity and arbitrary. It is clear that waves are excited with frequencics near the electron plasma frequency, and the maximal excited wave number agrees with theoretical one. The time history of the electric and magnetic-field energies are shown in Fig. 2. The electric field has a sharp rise at early times $\left(\omega_{p e} t<10\right)$, then reaches a saturation level. The magnetic field, however, changes little at early times, then increases after $\omega_{p e} t=10$. From these facts, we may draw the conclusion that the electron-beam instability drives the electrostatic waves which in turn generate electromagnetic waves.

\section{FORMATION OF ALFVÉN WAVES}

Figure 3 shows the phase space $z-v_{z}$ for the beam electrons, the background electrons and the positrons in the column at $\omega_{p e} t=10$. The particles are bunched fundamentally due to the excited electrostatic waves. The electrostatic mode is saturated because of the particle trapping in potential wells. The bunching of the particles has disappeared in the $z-v_{z}$ phase space after $\omega_{p e} t=25$. The time evolution of the magnetic field component $B_{x}$ along the beam axis is illustrated in Fig. 4. It is clear that the excited waves are propagating along the beam direction ( $z$ direc-
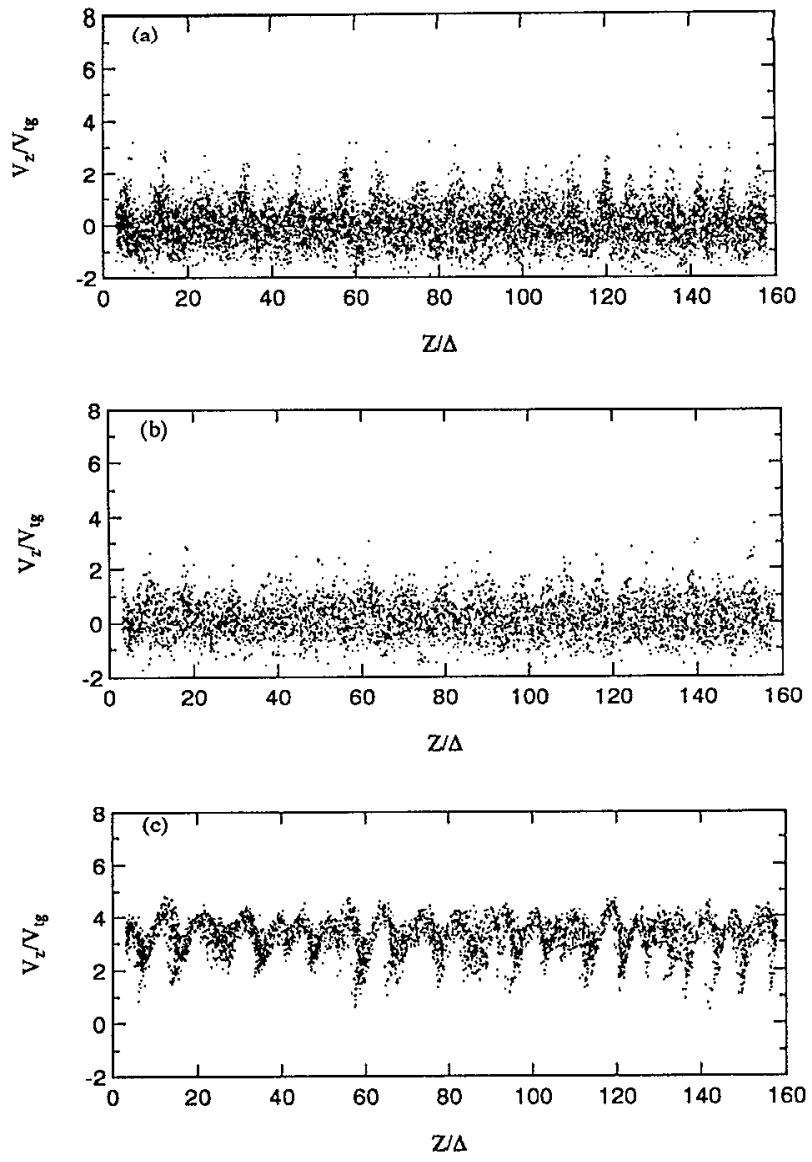

FIG. 3. The $z-v_{z}$ phase space distributions of particles in the column at $\omega_{p e} t=10$. (a) The positrons; (b) the background electrons; (c) the beam electrons.

tion). A spectrum of $B_{x}$ in $(\omega, k)$ inside the beam is shown in Fig. 5 using the same technique as for Fig. 1(b). The phase velocity estimated from Fig. 5 is of the order of $0.28 c$, where $c$ is the light velocity. The Alfvén velocity in an electron-positron plasma is given ${ }^{11}$ by $v_{\mathrm{A}} / c$ $=\left[1+2\left(\omega_{p e} / \omega_{c e}\right)^{2}\right]^{-1 / 2}$, or about $0.27 c$ for the simulation

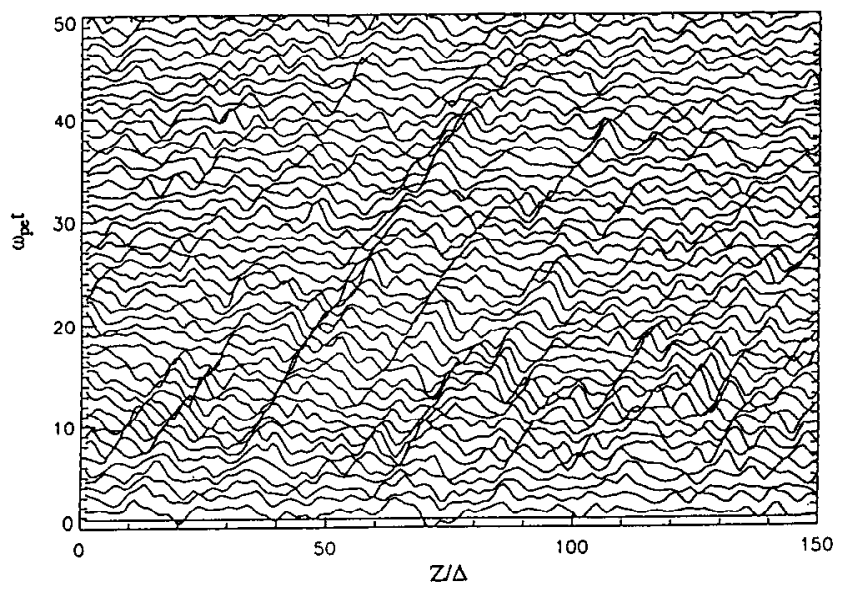

FIG. 4. The time evolution of $B_{x}$ along the $z$ direction in the center of beam. 


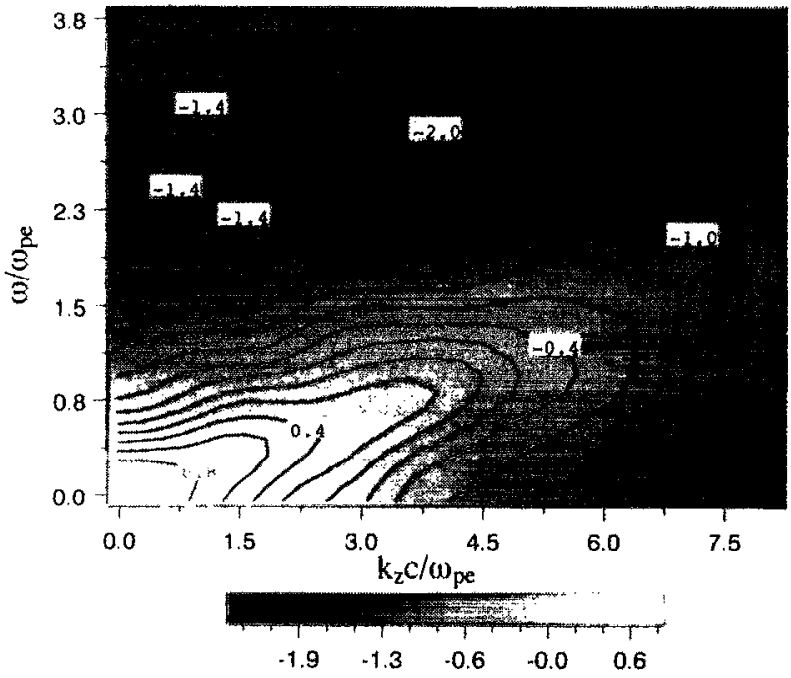

FIG. 5. Intensity of $B_{x}$ as a function of $\omega$ and $k_{z}$ along the $z$ direction in the center of beam.

parameters, which is nearly equal to the phase velocity. The excited electromagnetic waves show a right-hand polarization, as illustrated in Fig. 6, which plots the time history of $E_{x}$ vs $E_{y}$ obtained at the point $(x=43, y=43, z$ $=50$ ). Therefore, we infer that the excited electromagnetic mode is the Alfvén mode. The simulation results do not agree well with the amplitude ratio between the electric and magnetic fields obtained from the linear theory owing to nonlinear effects in the simulation. There is no evidence that the Alfven waves are excited before occurrence of the bunching at the time $\omega_{p e}<10$. From this fact, we reach the conclusion that the bunching of particles becomes the seed for the formation of Alfven waves. We will deduce this theoretically in the next part.

The basic equations ${ }^{1}$ describing the nonlinear evolution of waves in an electron-positron plasma in onedimensional (1-D) wave propagation along a constant magnetic field $B_{z 0}$ are

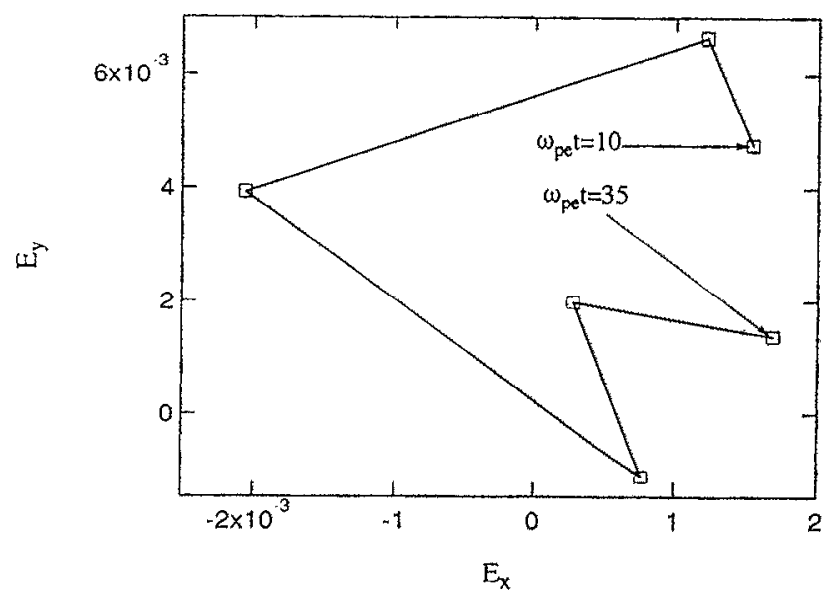

FIG. 6. The time history of $E_{x}$ vs $E_{y}$ taken at the point $(x=43, y=43$, $z=50$ ).

$$
\begin{aligned}
& m \frac{d v_{s}}{d t}=q\left(E-i \frac{B_{z 0}}{c} v_{s}+i \frac{v_{z}}{c} B\right), \\
& i \frac{\partial E}{\partial z}+\frac{1}{c} \frac{\partial B}{\partial t}=0, \\
& i\left(\frac{\partial^{2}}{\partial z^{2}}-\frac{1}{c^{2}} \frac{\partial^{2}}{\partial t^{2}}\right) B=\frac{4 \pi e n_{0}}{c} \frac{\partial}{\partial z}\left(v_{p}-v_{e}\right),
\end{aligned}
$$

where $d / d t=\partial / \partial t+v_{z} \partial / \partial z$, subscript $s$ denotes the species of particles ( $p$ and $e$ are the positrons and electrons), $v_{z}$ is the $z$ component of velocities, while the transverse components are given by $v_{s}=v_{s x}+i v_{s y}, B=B_{x}+i B_{y}, E=E_{x}+i E_{y}$. Now we introduce new coordinates

$$
\begin{aligned}
& \tilde{z}=z-v_{\mathrm{A}} t, \\
& \tilde{t}=t .
\end{aligned}
$$

With the coordinate transformation, we obtain the linear equations for the right-hand circular polarized mode from Eqs. (14)-(16) as follows:

$$
\begin{gathered}
\frac{4 \pi n_{0} e}{c} \frac{\partial}{\partial \widetilde{z}}\left(v_{p}-v_{e}\right)-i\left[\frac{\partial^{2}}{\partial \vec{z}^{2}}-\frac{1}{c^{2}}\left(\frac{\partial^{2}}{\partial \vec{t}^{2}}-2 v_{\mathrm{A}} \frac{\partial^{2}}{\partial \widetilde{z} \partial \widetilde{t}}\right.\right. \\
\left.\left.+v_{\mathrm{A}}^{2} \frac{\partial^{2}}{\partial \widetilde{z}^{2}}\right)\right] B=0, \\
\left(\frac{\partial^{2}}{\partial \widetilde{z} \partial \bar{t}}-v_{\mathrm{A}}^{2} \frac{\partial^{2}}{\partial \bar{z}^{2}}\right) v_{p}+\frac{\partial v_{z}}{\partial \widetilde{z}} \frac{\partial v_{p}}{\partial \widetilde{z}}+i \omega_{c} \frac{\partial v_{p}}{\partial \widetilde{z}} \\
\quad-\frac{i e}{m c}\left(\frac{\partial B}{\partial \widetilde{t}}-v_{\mathrm{A}} \frac{\partial B}{\partial \bar{z}}+B \frac{\partial v_{z}}{\partial \widetilde{z}}\right)=0, \\
\left(\frac{\partial^{2}}{\partial \widetilde{z} \partial \tilde{t}}-v_{\mathrm{A}}^{2} \frac{\partial^{2}}{\partial \widetilde{z}}\right) v_{e}+\frac{\partial v_{z}}{\partial \widetilde{z}} \frac{\partial v_{e}}{\partial \widetilde{z}}+i \omega_{c} \frac{\partial v_{e}}{\partial \widetilde{z}} \\
+\frac{i e}{m c}\left(\frac{\partial B}{\partial \widetilde{t}}-v_{\mathrm{A}} \frac{\partial B}{\partial \widetilde{z}}+B \frac{\partial v_{z}}{\partial \widetilde{z}}\right)=0,
\end{gathered}
$$

where $\omega_{c}=e B_{z 0} / m c$ is the cyclotron frequency. On the other hand, the left-hand circular polarized mode is obtained by taking the complex conjugate. To obtain exact dispersion relation, it requires eigenmode analysis which is very complicated. In order to overcome this difficulty, Wentzel-Kramers-Brillouin (WKB) approximation method can be applied to derive the dispersion relation with validity condition $k L_{0}>1$, where $k$ and $L_{0}$ are the wave number and the characteristic length. Taking $k=1.8 \omega_{p e} / c$ as the excited wave number at the region where the Alfvén waves are strongly excited and $L_{0}=5.7 \Delta$ as the scale length of bunches in the simulation, the above value $k L_{0}$ is about 2 which roughly satisfies the condition. The same dispersion relation for both $R$ mode and $L$ mode is given by

$$
\begin{gathered}
{\left[k^{2} c^{2}+2 \omega_{p e}^{2}-\left(\omega+v_{\mathrm{A}} k\right)^{2}\right]\left(\omega+v_{\mathrm{A}} k+i a\right)^{2}} \\
-\omega_{c}^{2}\left[k^{2} c^{2}-\left(\omega+v_{\mathrm{A}} k\right)^{2}\right]=0,
\end{gathered}
$$

where $a=\partial v_{z} / \partial \tilde{z}$. In the above dispersion relation, there are two modes: high-frequency electromagnetic waves and 

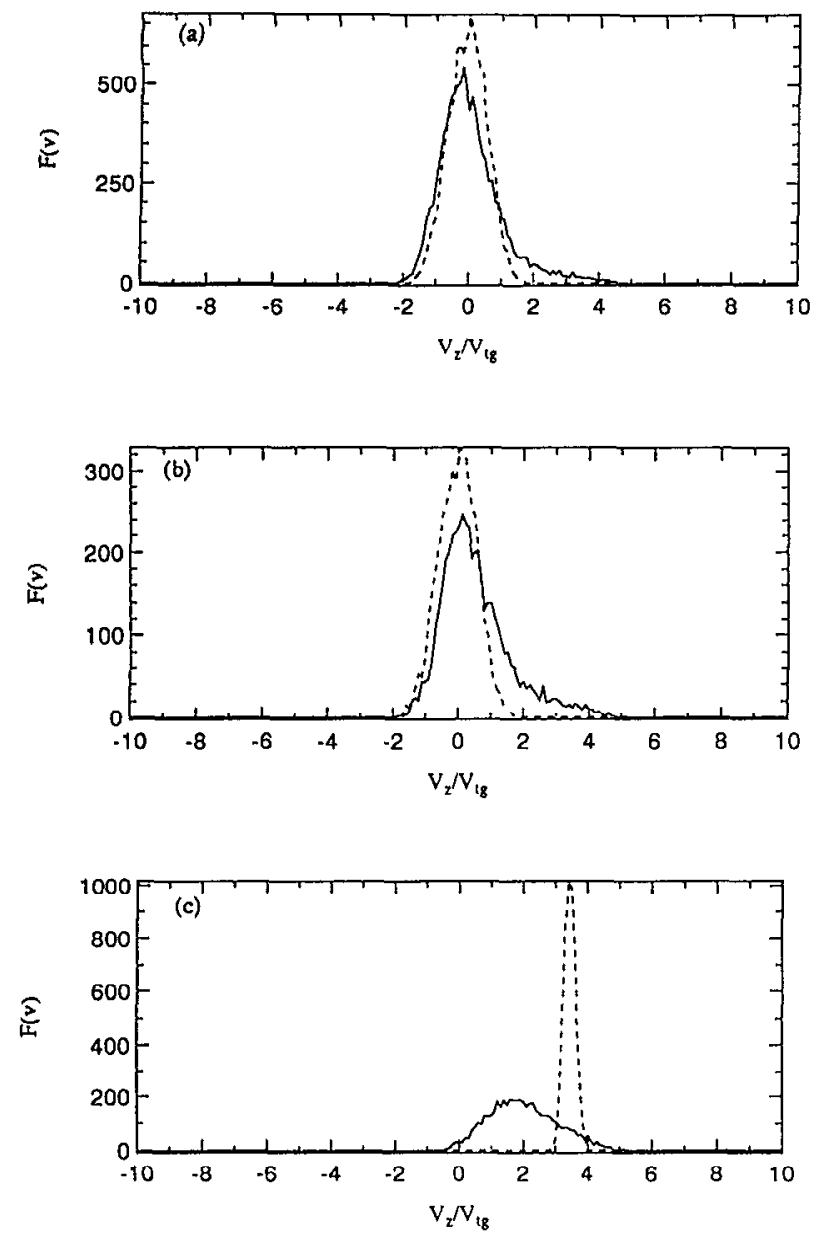

FIG. 7. The velocity distributions of particles in the column along the $z$ direction at $t=0$ (dashed line) and $\omega_{p e} t=20$ (full line). (a) The positrons; (b) the background electrons; (c) the beam electrons.

low-frequency Alfven waves. In the region of the Alfven waves, substituting $\omega=\omega_{r}+i \omega_{i}$ and solving the Eq. (22), it yields

$$
\omega_{i}=-a\left(k^{2} c^{2}+2 \omega_{p e}^{2}\right) /\left(k^{2} c^{2}+2 \omega_{p e}^{2}+\omega_{c}^{2}\right) .
$$

The condition $\partial v_{z} / \partial \widetilde{z}<0, \omega_{i}>0$ correspond to a growing unstable mode. The simulation results show that particles are bunched along the $z$ direction, accelerated and decelerated, which means that $\partial v_{z} / \partial \widetilde{z}$ generally is not equal to zero, and that region exists where waves should be generated. We approximately estimate $\omega_{i}=-a$ in the limit of $\left(k c / \omega_{p e}\right)^{2}<1$. The growth time is of the order of $4 \omega_{p e}^{-1}$ consistent well with the simulation results. So it infers that the Alfven waves are excited due to occurrence of the bunches.

\section{PARTICLE ACCELERATION BY SOLITARY ALFVÉN WAVES}

Sakai and Kawata ${ }^{1}$ and Mikhailovski et $a l^{2}$ pointed out that the modified Kortweg-de Vries equation which is used to describe the characteristics of Alfvén waves has a soliton solution in electron-positron plasmas. The $\omega-k$ spectrum shown in Fig. 5 is remarkably similar to the spec- trum of damped solitary waves propagating with the same velocity, as found in the simulation. From this fact, we conclude that the Alfven waves excited from the strong bunching regions propagate as damped solitary waves. Figure 7 plots the velocity distributions of background electrons, positrons, and beam electrons at $t=0$ (dashed line) and $t=20 \omega_{p e} t$ (full line). The background electrons and positrons are accelerated by the beam mode before excitation of the Alfven waves (see Fig. 3). When the beam mode becomes saturated, the particles continue to be accelerated by the Alfvén waves. The acceleration is restrained after disappearance of the waves. The beam electrons are mostly decelerated during all these procedures.

In order to interpret the acceleration mechanism, we investigate the motion of a test particle (electron and positron) in perpendicular components of electric and magnetic fields of Alfvén waves propagating along the $z$ direction. Our simulation results show that the magnetic component in the $x$ direction is dominant compared with the one in the $y$ direction. We assume magnetic field $B=\left(B_{x}, 0, B_{z 0}\right)$ and electric field $E=\left(0, E_{y}, E_{z}\right)$, where $E_{z}$ associates with the saturated electrostatic component. As the damped solitary waves are found to be excited, $B_{x}$ is given by the soliton solution, $B_{x}=e^{-a t} / \cosh \left[\left(z-v_{p} t\right) / \lambda\right]$. From Maxwell equations, it is easy to calculate $E_{y}$ $=-a e^{-a t} \lambda \operatorname{arc}\left(\sin \left\{\tanh \left[\left(z-v_{p} t\right) / \lambda\right]\right\}\right)-v_{p} e^{-a t} \cosh [(z$ $\left.-v_{p} t\right) / \lambda$ ]. Normalized equations for the test particle are given as follows:

$$
\frac{d z}{d t}=v_{t g} \omega_{p e}^{-1} p_{z}
$$

$$
\frac{d p_{x}}{d t}=\frac{\omega_{c e}}{\omega_{p e}}\left(p_{y} B_{z 0}\right)
$$

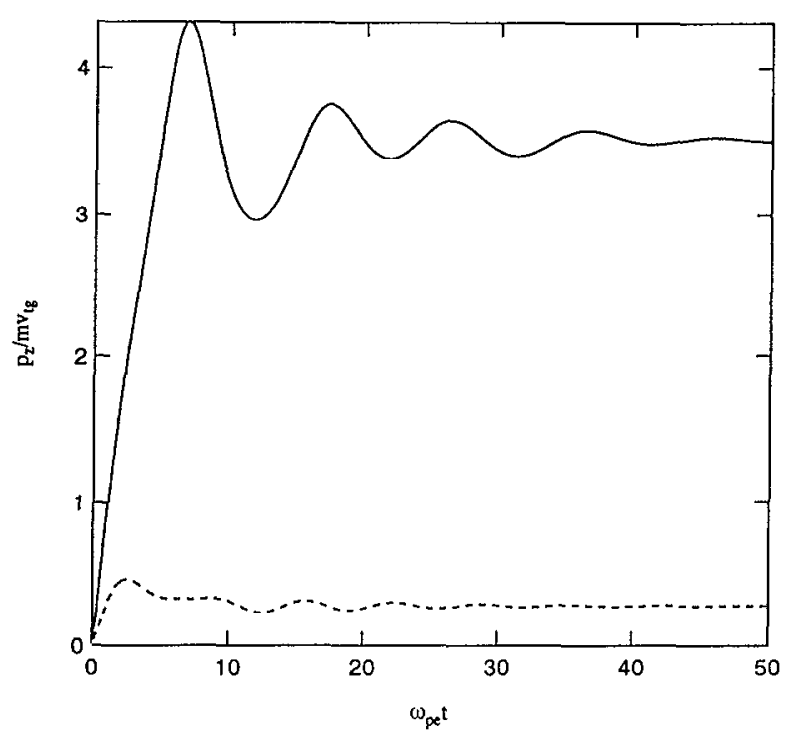

FIG. 8. The time evolutions of the test electron momentum in the $z$ direction for one soliton (dashed line) and eight solitons (full line) propagating with the same initial conditions as $p_{x}=p_{y}=p_{z}=0, z=88$. 

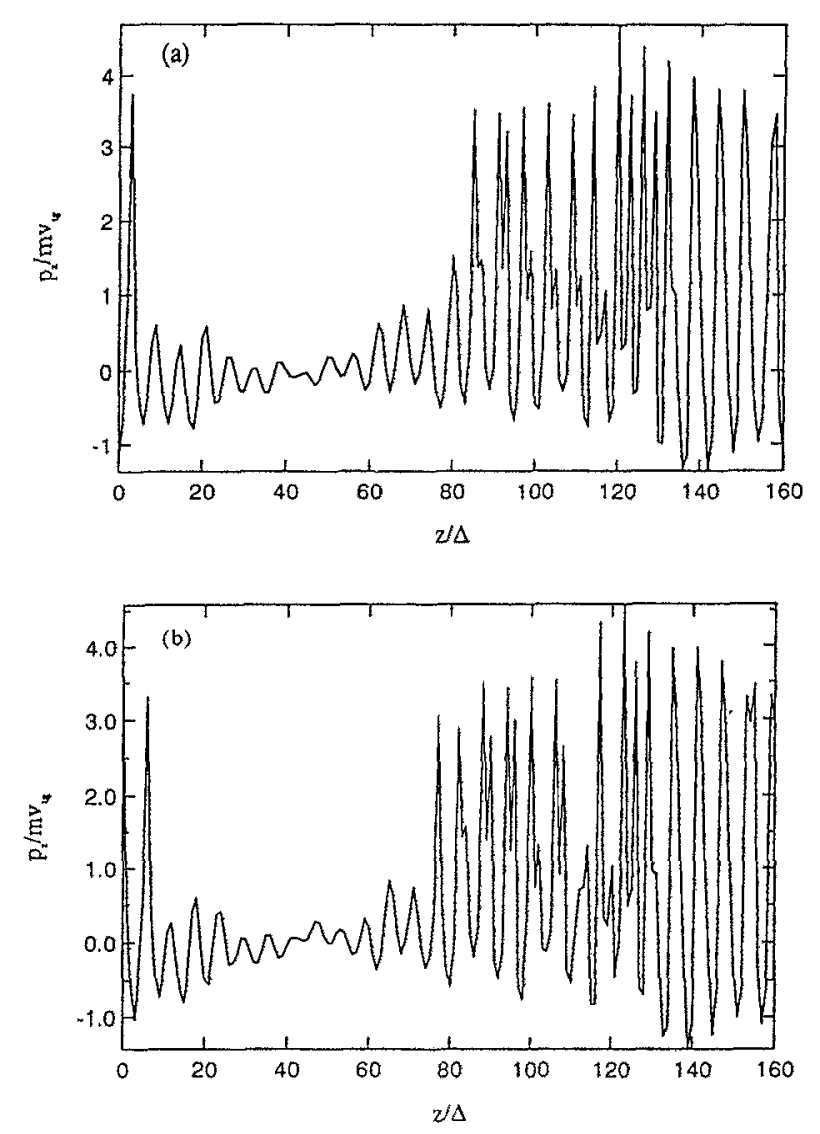

FIG. 9. The momenta in the $z$ direction at $\omega_{p e} t=50$ for different initial positions of the particle with the same initial momenta as $p_{x}=p_{y}=p_{z}=0$. (a) The positron; (b) the electron.

$$
\begin{aligned}
& \frac{d p_{y}}{d t}=\frac{\omega_{c e}}{v_{t g}} E_{y}+\frac{\omega_{c e}}{\omega_{p e}}\left(p_{z} B_{x}-p_{x} B_{z 0}\right), \\
& \frac{d p_{z}}{d t}=\frac{\omega_{c e}}{v_{t g}} E_{z}-\frac{\omega_{c e}}{\omega_{p e}} p_{y} B_{x},
\end{aligned}
$$

where $p$ is the momentum of the particle. Time, $z, p, B, E$ are normalized by $\omega_{p e}^{-1}, \Delta, m v_{t g}, B_{z 0}, B_{z 0} \Delta / \omega_{p e}$, respectively. The numerical results show that the particle can be accelerated along the beam direction and oscillates in the perpendicular directions with nearly same characteristics for both electron and positron. Figure 8 shows of the time evolutions of the electron momentum in the $z$ direction for one soliton and eight solitons propagating with the same parameters as used in the particle simulation. It is clear that many solitons, which are found propagating simultaneously in the particle simulation results, are able to accelerate the particles up to the energy level obtained in the simulation. Figure 9 illustrates how the acceleration de- pends on initial positions with the same initial momenta $P_{x}=P_{y}=P_{z}=0$. The final energies agree quite well with the simulation results shown in Fig. 7. Based on the results of particle simulation and test particle calculation, we conclude that the Alfven waves are important energy converted from the electron beam to accelerate the background particles.

\section{CONCLUSION}

We have presented results of electrostatic waves driven by the beam instability and the formation of Alfvén waves from a 3-D EM particle simulation of an electron-positron plasma. It has been found that the beam instability creates particle bunching in the plasma. The simulation results have shown that particle bunching appears prior to the excitation of Alfvén waves, and a theoretical analysis has shown that Alfvén waves are generated by these bunches. The Alfren waves excited from the strong bunching regions propagate as damped solitons and accelerate the background electrons and positrons.

\section{ACKNOWLEDGMENTS}

The authors wish to acknowledge contributions from Professor Oscar Buneman for development of the code. We greatly appreciate the thoughtful comments and suggestions of Dr. S. Koide, Dr. B. Chargeishvil, and the referee.

This work is sponsored by the U.S.-Japan cooperative science program of Japan Society for the Promotion of Science and National Science Foundation (NSF) (INT9217650). J. Zhao is grateful to the Japanese Education Ministry for scholarship. K.-I. Nishikawa is supported by NSF under Grant Nos. ATM-9106639, ATM-9119814, and ATM-912116. T. Neubert is supported by National Aeronautics and Space Administration under Contract No. NASA-35350.

IJ.-I. Sakai and T. Kawata, J. Phys. Soc. Jpn. 49, 753 (1980).

${ }^{2}$ A. B. Mikhailovskii, O. G. Onishchenko, and A. I. Smolyakov, Sov, J. Plasma Phys. 11, 215 (1985).

${ }^{3}$ L. Stenflo, P. K. Shukla, and M. Y. Yu, Astro. Space Sci. 117, 303 (1985).

${ }^{4}$ M. Y. Yu, K. Shukla, and L. Stenflo, Astrophys. J. 309, 63 (1986).

${ }^{5}$ R. Bharuthram, P. K. Shukla, and M. Y. Yu, Astro. Space Sci. 135, 211 (1987).

${ }^{6}$ O. Buneman, in Computer Space Plasma Physics, Simulation Techniques and Softwares, edited by H. Matsumoto and Y. Omura (Terra Scientific, Tokyo, 1993), p. 1 .

${ }^{7} \mathrm{O}$. Buneman, T. Neubert, and K.-I. Nishikawa, IEEE Trans. Plasma Sci, PS-20, 810 (1992).

${ }^{8}$ T. Neubert, R. H. Miller, O. Buneman, and K.-I. Nishikawa, Geophys. Res. 97, 12057 (1992).

${ }^{9}$ K.-I. Nishikawa, O. Buneman, and T. Neubert, "New aspects of whistler waves driven by an electron beam studied by a 3-d electromagnetic code," submitted to Geophys. Res. Lett. (1992).

${ }^{10}$ E. L. Lindman, J. Comput. Phys. 18, 66 (1975).

"J.-I. Sakai and T. Kawata, J. Phys. Soc. Jpn. 49, 747 (1980). 